\title{
INTERFACE SEPARATION CAUSED BY A PLANE ELASTIC WAVE OF ARBITRARY FORM
}

\author{
J. DUNDURS \\ Department of Civil Engineering Northwestern University, Evanston, IL, U.S.A. \\ Maria COMNINOU \\ Department of Applied Mechanics and Engineering Science University of Michigan, Ann Arbor, MI, U.S.A.
}

Received January 9, 1978

\begin{abstract}
The paper considers the separation between two contacting solids caused by an incident elastic wave. The wave is assumed to be plane, but may have an arbitrary form. The unilateral interface between the solids is taken as frictionless and incapable of transmitting tension. If the disturbance propagates along the interface with a speed that is supersonic with respect to both solids, the problem can be solved in closed form, and simple results for the extent of the separation zones and the respective gaps are obtained. Several specific examples are included.
\end{abstract}

\section{Introduction}

The reflection and refraction of elastic waves is customarily discussed on the basis of a bonded or a bilateral interface: all field quantities entering the boundary conditions are taken as continuous at the interface, and the resulting relations are in the form of equalities. In contrast, a unilateral interface allows certain discontinuities, and the boundary conditions also involve inequalities. Consider, for instance, two solids that are in contact, and assume that they are not bonded to each other. If an incident wave strikes such an interface, localized separation and slip can take place resulting in displacement discontinuities. Moreover, inequalities must be incorporated in the boundary conditions to ensure that the normal tractions are not tensile, and that there is no overlapping of material.

We treated recently several aspects of plane harmonic waves interacting with a frictionless interface that permits separation [1, 2]. Such steadily maintained harmonic waves lead to a periodic array of separation and contact zones, and the problem could be solved by taking advantage of the periodicity. The present paper considers a planc wave of an arbitrary form that may result in an irregular array of separation and contact zones running along the interface.

Suppose that an elastic wave, such as a stress pulse with a plane front and phase velocity $c_{0}$ strikes the interface under the angle $\theta_{0}$ (angle between the normal to the interface and the direction of propagation of the incident pulse). Then the point of intersection of the wave front and the interface propagates with the velocity

$$
v=c_{0} / \sin \theta_{u}
$$

The mathematical nature of the problem depends drastically on the range of the trace velocity $v$. If $v$ is above the largest velocity of longitudinal waves in the two solids, a bilateral interface reflects and refracts a wave of the $\mathrm{P}$ - and SV-type in the same manner as harmonic waves. In particular, the reflected and refracted waves are simply proportional to the incident wave. If $v$ falls below the largest velocity of longitudinal waves, however, 
the problem becomes considerably more complicated [3]. The situation is also quite similar with the unilateral interface that separates: For $v$ in the purely supersonic range, the problem can be solved in closed form. In other cases, the formulation leads to singular integral equations. The present investigation is restricted to the supersonic case, and the purpose of this paper is to show how to correct the bilateral solution by cancelling the tensile tractions and how to find the resulting gaps between the solids.

\section{Formulation}

We consider two elastic solids that are held together by the applied pressure $p^{\infty}$. An incident plane $\mathrm{P}$ - or SV-wave of arbitrary form strikes the frictionless interface under the angle $\theta_{0}$ and is reflected and refracted as indicated in Fig. 1. The indices $n(=0,1,2,3,4)$ are used to distinguish

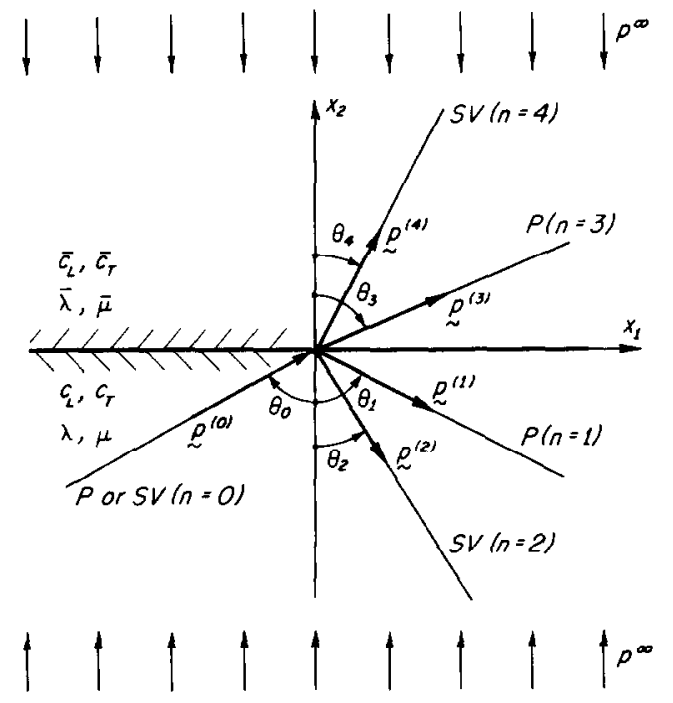

Fig. 1. Incident $(n=0)$, reflected $(n=1,2)$ and refracted $(n=$ 3,4 ) waves.

between the various waves. Bars will be used to refer to the material constants and other quantities pertaining to the upper solid.
The displacement of the incident wave is taken as

$$
u^{(0)}=C_{0} d^{(0)} F\left(y_{0}\right)
$$

where

$$
y_{0}=k_{0}\left[\boldsymbol{x} \cdot \boldsymbol{p}^{(0)}-c_{0} t\right]
$$

Furthermore, $d^{(0)}$ and $p^{(0)}$ are unit vectors defining the directions of motion and propagation [4], and $C_{0}$ is taken as real. Suppose at first that the frictionless interface is bilateral, viz. tensile tractions are permitted and the normal displacements are continuous. The reflected and refracted waves are then of the same form as (2), except that the vector $\boldsymbol{d}$ and the argument $y$ of the function $F$ must be appropriately modified. The amplitudes of the reflected and refracted waves are determined by the reflection and transmission coefficients for a frictionless interface given in [1]. Of interest for our purposes are only the normal tractions transmitted by the interface [1]:

$$
\begin{aligned}
S(\eta) & =\left[\sigma_{22}^{(0)}+\sigma_{22}^{(1)}+\sigma_{22}^{(2)}\right]_{x_{2}=0} \\
& =\left[\sigma_{22}^{(3)}+\sigma_{22}^{(4)}\right]_{x_{2}=0} \\
& =\mathscr{A}_{0} f(\eta)
\end{aligned}
$$

where

$$
\eta=k_{0}\left[x_{1} p_{1}^{(0)}-c_{0} t\right]
$$

is a coordinate moving with the velocity $c_{0} / \sin \theta_{0}$, and

$$
f(\eta)=\left.\frac{\mathrm{d} F\left(y_{0}\right)}{\mathrm{d} y_{0}}\right|_{x_{2}=0}
$$

With abbreviations given below,

$$
\mathscr{A}_{0}=C_{0} k_{2} \mu\left(\Omega \Gamma \gamma^{-1} \bar{q} \cos \theta_{1}\right)
$$

for an incident P-wave $\left(\theta_{1}=\theta_{0}\right)$, and

$$
\mathscr{A}_{0}=C_{0} k_{1} \mu \tan 2 \theta_{2}\left(\Omega \Gamma \gamma^{-1} \bar{q} \cos \theta_{1}\right)
$$

for an incident SV-wave $\left(\theta_{2}=\theta_{0}\right)$. The contractions used are

$$
\begin{array}{ll}
\kappa=c_{\mathrm{L}} / c_{\mathrm{T}}, & \bar{\kappa}=\bar{c}_{\mathrm{L}} / \bar{c}_{\mathrm{T}} ; \\
\gamma=\bar{c}_{\mathrm{L}} / c_{\mathrm{L}}, & \Gamma=\bar{\mu} / \mu ;
\end{array}
$$




$$
\begin{aligned}
& q=\sin 2 \theta_{1} \sin 2 \theta_{2}+\kappa^{2} \cos ^{2} 2 \theta_{2} ; \\
& \bar{q}=\sin 2 \theta_{3} \sin 2 \theta_{4}+\bar{\kappa}^{2} \cos ^{2} 2 \theta_{4} ; \\
& \Omega=\frac{2 \kappa \cos 2 \theta_{2}}{q \cos \theta_{3}+\Gamma \gamma^{-1} \bar{q} \cos \theta_{1}}
\end{aligned}
$$

where $c_{\mathrm{L}}$ and $c_{\mathrm{T}}$ denote the phase velocities of longitudinal and transverse waves. Furthermore,

$$
\begin{aligned}
& k_{0} c_{0}=k_{1} c_{\mathcal{L}}=k_{2} c_{\mathrm{T}}=k_{3} \bar{c}_{\mathrm{L}}=k_{4} \bar{c}_{\mathrm{T}} \\
& \frac{\sin \theta_{0}}{c_{0}}=\frac{\sin \theta_{1}}{c_{\mathrm{L}}}=\frac{\sin \theta_{2}}{c_{\mathrm{T}}}=\frac{\sin \theta_{3}}{\bar{c}_{\mathrm{L}}}=\frac{\sin \theta_{4}}{\bar{c}_{\mathrm{T}}} .
\end{aligned}
$$

It is readily seen from (7) and (8) that $\mathscr{A}_{0}$ is of the same sign as $C_{0}$.

We assume that the interface tractions in the bilateral problem given by (4) contain some tension zones. The next step then is to correct the bilateral solution by cancelling the tensile tractions, while allowing localized separation at the interface. This problem could conceivably be approached in a variety of ways. We prefer to view the situation in terms of moving dislocations. A separation zone propagating along the interface is in fact nothing else but an array of distributed edge dislocations that move by climb: the Burgers vectors of the dislocations are perpendicular to the interface and their extra sheets lie in the interface. If $g(\eta)$ denotes the gap (separation distance measured normal to the interface), the density of the distributed dislocations is [7]

$$
B(\eta)=-\frac{\mathrm{d} g(\eta)}{\mathrm{d} \eta}
$$

Distributed edge dislocations that move along a bonded interface have been treated by Berg et al. [5]. We need, however, the corresponding results for a frictionless interface that is free to slip.

An array of edge dislocations propagating along the interface with a speed that falls in the supersonic range with respect to both materials emits $P$ and $S V$-waves in both solids [6,7]. Moreover, each of the four waves can be considered separately and related to a displacement discontinuity at the interface. Thus we consider the total dislocation distribution $B(\eta)$ as consisting of the four parts
$B_{\mathrm{L}}, B_{\mathrm{T}}, \bar{B}_{\mathrm{L}}, \bar{B}_{\mathrm{T}}$ associated with the longitudinal and transverse waves in the lower and upper solids, respectively, so that

$$
B(\eta)=B_{\mathrm{I}}(\eta)+B_{\mathrm{T}}(\eta)+\bar{B}_{\mathrm{L}}(\eta)+\bar{B}_{\mathrm{T}}(\eta) .
$$

The interface tractions produced by each of the distributions are given by Berg et al. [5]. Thus

$$
\begin{aligned}
& \tau_{x y}(\eta, 0)=\mu\left[2 B_{\mathrm{L}}(\eta)-\left(\zeta_{\mathrm{T}}^{2}-1\right) B_{\mathrm{T}}(\eta)\right], \\
& \tau_{y y}(\eta, 0)=-\mu\left[\zeta_{\mathrm{L}}^{-1}\left(\zeta_{\mathrm{T}}^{2}-1\right) B_{\mathrm{L}}(\eta)+2 \zeta_{\mathrm{T}} B_{\mathrm{T}}(\eta)\right]
\end{aligned}
$$

in the lower solid, and

$$
\begin{gathered}
\bar{\tau}_{x y}(\eta, 0)=\bar{\mu}\left[-2 \bar{B}_{\mathrm{L}}(\eta)+\left(\bar{\zeta}_{\mathrm{T}}^{2}-1\right) \bar{B}_{\mathrm{T}}(\eta)\right], \\
\bar{\tau}_{y y}(\eta, 0)=-\bar{\mu}\left[\bar{\zeta}_{\mathrm{L}}^{-1}\left(\bar{\zeta}_{\mathrm{T}}^{2}-1\right) \bar{B}_{\mathrm{L}}(\eta)\right. \\
\left.+2 \bar{\zeta}_{\mathrm{T}} \bar{B}_{\mathrm{T}}(\eta)\right]
\end{gathered}
$$

in the upper solid. In these equations,

$$
\zeta_{\mathrm{L}}=\left(\frac{v^{2}}{c_{\mathrm{L}}^{2}}-1\right)^{1 / 2}, \quad \zeta_{\mathrm{T}}=\left(\frac{v^{2}}{c_{\mathrm{T}}^{2}}-1\right)^{1 / 2}
$$

with analogous expressions being valid for $\bar{\zeta}_{\mathrm{L}}$ and $\bar{\zeta}_{\mathrm{T}}$.

The four distributions are computed in terms if $B(\eta)$ from the boundary conditions at the interface. We require that the normal tractions be continuous and the shearing tractions vanish. Thus, three equations in addition to (16) are available. Solving the system simultaneously yields

$$
\begin{aligned}
& B_{\mathrm{L}}(\eta)=\frac{1}{2}\left(\zeta_{\mathrm{T}}^{2}-1\right) B_{\mathrm{T}}(\eta), \\
& \bar{B}_{\mathrm{L}}(\eta)=\frac{1}{2}\left(\bar{\zeta}_{\mathrm{T}}^{2}-1\right) \bar{B}_{\mathrm{T}}(\eta), \\
& B_{\mathrm{T}}(\eta)=\frac{2 \Gamma \zeta_{\mathrm{L}} \bar{R}}{\Delta} B(\eta), \\
& \bar{B}_{\mathrm{T}}(\eta)=\frac{2 \bar{\zeta}_{\mathrm{L}} R}{\Delta} B(\eta)
\end{aligned}
$$

where

$$
\begin{aligned}
& R=\left(\zeta_{\mathrm{T}}^{2}+1\right)^{2}+4 \zeta_{\mathrm{L}} \zeta_{\mathrm{T}}, \\
& \bar{R}=\left(\bar{\zeta}_{\mathrm{T}}^{2}+1\right)^{2}+4 \bar{\zeta}_{\mathrm{L}} \bar{\zeta}_{\mathrm{T}}, \\
& \Delta=\Gamma_{\zeta_{\mathrm{L}}}\left(\zeta_{\mathrm{T}}^{2}+1\right) \bar{R}+\bar{\zeta}_{\mathrm{L}}\left(\bar{\zeta}_{\mathrm{T}}^{2}+1\right) R .
\end{aligned}
$$


The normal tractions at the interface induced by the distribution of dislocations moving with the velocity $v$ are from (18) or (20)

$$
T(\eta)=\tau_{y y}(\eta, 0)=\bar{\tau}_{y y}(\eta, 0)=-\frac{\bar{\mu} R \bar{R}}{\Delta} B(\eta)
$$

Using (9-14), this expression can also be put in the form

$$
T(\eta)=-\mathscr{A}_{d} B(\eta)
$$

where

$$
\mathscr{A}_{d}=\frac{\bar{\mu} q \bar{q} \Omega}{2 \kappa \gamma \sin \theta_{1} \cos 2 \theta_{2}}>0 .
$$

The total normal tractions transmitted by the interface are

$$
N(\eta)-S(\eta)-p^{\infty}+T(\eta)
$$

The first two terms in this expression are the interface tractions in the bilateral problem with precompression; the last term may be viewed as a correction which accounts for the separation between the solids.

The conditions the unilateral solution must satisfy are

$$
N(\eta)=0, \quad g(\eta) \geqslant 0
$$

in the separation zones, and

$$
g(\eta)=0, \quad N(\eta) \leqslant 0
$$

in the contact zones. Denoting a typical separation interval by $(\alpha, \beta)$, and substituting (4) and (27) into (30) we obtain

$$
B(\eta)=\frac{\mathscr{A}_{0}}{\mathscr{A}_{d}}\left[f(\eta)-\frac{p^{\infty}}{\mathscr{A}_{0}}\right] \quad(\alpha<\eta<\beta) .
$$

The unknowns $\alpha$ and $\beta$ must be determined so as to satisfy the inequalities (31) and (33). Moreover, if the gap is to close, we must also have

$$
\int_{\alpha}^{\beta} B(\eta) \mathrm{d} \eta=0
$$

\section{Solution}

The remaining task is to find the endpoints of the separation zones and to determine the resulting gaps. We observe toward this end the following:

(i) As seen from (34), the local value of the dislocation density $B(\eta)$ is detcrmined solcly by the local value of the interface tractions in the bilateral problem. It follows from (27) and (29) that, conversely, the contribution of the dislocation distribution to the interface tractions is purely local. This is a consequence of the fact that the dislocations move with a supersonic velocity.

(ii) The dislocation density $B(\eta)$ and therefore also the corrective tractions $T(\eta)$ from (27) vanish in the contact zones. Consequently the interface tractions in the contact zones are the same as in the bilateral problem. In other words, the dislocations erase the interface tractions in the separation zones, but leave them unaffected elsewhere.

(iii) As seen from (15), (27) and (28), $\mathrm{d} g(\eta) / \mathrm{d} \eta$ and $T(\eta)$ are of the same algebraic sign.

It is clear that for $0<\theta_{0}<\frac{1}{2} \pi$, the tension zones in the bilateral problem and thus the separation intervals propagate to the right. Considering again a typical separation interval $(\alpha, \beta)$ in the moving coordinate $\eta$, the leading edge of the separation zone is $\eta=\beta$ and the trailing edge $\eta=\alpha(\alpha<\beta)$. We can reason now that the leading edge of a separation zone coincides with the leading edge of a tension zone in the bilateral problem with precompression. Let us take the leading edge of the tension zone at $\eta=\beta^{*}$. Suppose first that $\beta<\beta^{*}$ so that a contact zone extends inside the tension zone. Then by (ii), $T(\eta)=0$ and consequently $N(\eta)>0$ in the interval $\beta<\eta<\beta^{*}$ which violates (33). Suppose next that $\beta>\beta^{*}$, so that the leading edge of the separation interval falls inside a compression region of the bilateral problem. Then $T(\eta)>0$ in the interval $\beta^{*}<\eta<\beta$. Since $g(\beta)=0$, we have

$$
g(\eta)=\int_{\beta}^{\eta} \frac{\mathrm{d} g(\xi)}{\mathrm{d} \xi} \mathrm{d} \xi .
$$

According to (iii), $\mathrm{d} g(\eta) / \mathrm{d} \eta>0$ and (36) will yield 
$g(\eta)<0$ in $\beta^{*}<\eta<\beta$ because the integration proceeds in the negative direction. Thus (31) is violated. Consequently $\beta=\beta^{*}$, and it follows that $\beta$ is determined from

$$
S(\beta)-p^{\infty}=\mathscr{A}_{0} f(\beta)-p^{\infty}=0
$$

with the understanding that the root corresponds to the leading edge of the tension zone.

It may be noted that the trailing edge $\eta=\alpha$ of the separation interval cannot be found by using similar arguments. On basis of (ii) we can claim that it is impossible for $\alpha$ to fall inside a tension zone. However, no contradiction is reached considering the sign of $\mathrm{d} g(\eta) / \mathrm{d} \eta$ when $\alpha$ falls in a compression zone.

Substituting (15) and (34) into (36) we get the gap

$$
g(\eta)=\frac{\mathscr{A}_{0}}{\mathscr{A}_{d}}\left\{\int_{\eta}^{\beta} f(\xi) \mathrm{d} \xi-\frac{p^{\infty}}{\mathscr{A}_{0}}(\beta-\eta)\right\} .
$$

The trailing edge of the separation interval is fixed by the requirement that $g(\alpha)=0$. Therefore $\alpha$ is determined by the condition

$$
\frac{p^{\infty}}{\mathscr{A}_{0}}(\beta-\alpha)=\int_{\alpha}^{\beta} f(\xi) \mathrm{d} \xi
$$

which is also equivalent to (35).

Once one of the separation intervals has been established, it is possible to treat by the same means the next zone on the left. The only remaining question might be how to get the process started because a single separation zone can conceivably cover several tension zones. There is no difficulty, however, if the incident wave has a well defined front, and we shall not discuss this question. We also refrain from constructing the full elastic fields in the solids. Since the dislocation density $B(\eta)$ is known from (34), this can be done quite simply as explained by Weertman in his study of supersonic edge dislocations [6].

\section{Examples}

In order to illustrate the general results and to gain some additional insight we consider a few simple examples.

\subsection{Parabolic tension pulse}

We take

$$
f(\eta)= \begin{cases}1-\eta^{2} & (|\eta| \leqslant 1), \\ 0 ; & (|\eta| \geqslant 1) .\end{cases}
$$

The incident wave is thus assumed to induce a single tension zone of a particularly simple form. From (37), the leading edge of the separation zone is specified by

$$
\beta=\left(1-p^{\infty} / \mathscr{A}_{0}\right)^{1 / 2}, \quad p^{\infty} / \mathscr{A}_{0} \leqslant 1 .
$$

In applying (38) and (39), however, we must distinguish between two cases depending on the level of the applied pressure $p^{\infty}$. At higher levels of the applied pressure, the separation zone is short and $\alpha>-1$. In such a case

$$
g(\eta)=\frac{\mathscr{A}_{0}}{3 \mathscr{A}_{d}}(\beta-\eta)^{2}(2 \beta+\eta) \quad(-1 \leqslant \alpha \leqslant \eta \leqslant \beta)
$$

and

$$
\alpha=-2 \beta
$$

These results are valid for

$$
\frac{3}{4} \leqslant \frac{p^{\infty}}{\mathscr{A}_{0}} \leqslant 1
$$

If the applied pressure is less than specified by (44), the separation zone penetrates on the left into a region that would be totally unaffected by the finite pulse in the bilateral problem. Thus for

$$
0 \leqslant \frac{p^{\infty}}{\mathscr{A}_{0}} \leqslant \frac{3}{4}
$$

we get

$$
g(\eta)=\frac{\mathscr{A}_{0}}{3 \mathscr{A}_{d}}\left\{\begin{array}{l}
(\beta-\eta)^{2}(2 \beta+\eta) \\
(-1 \leqslant \eta \leqslant \beta), \\
(1+\beta)\left[2\left(1-\beta+\beta^{2}\right)+3(1-\beta) \eta\right] \\
(\alpha \leqslant \eta \leqslant-1)
\end{array}\right.
$$

and

$$
\alpha=-\frac{2\left(1-\beta+\beta^{2}\right)}{3(1-\beta)}
$$

where $\beta$ is still given by (41). 
The coordinates $\alpha$ and $\beta$ determining the extent of the separation zone are given in terms of $p^{\infty} / \mathscr{A}_{0}$ in Fig. 2. It is seen that the separation zone at relatively low levels of the applied pressure $p^{\infty}$

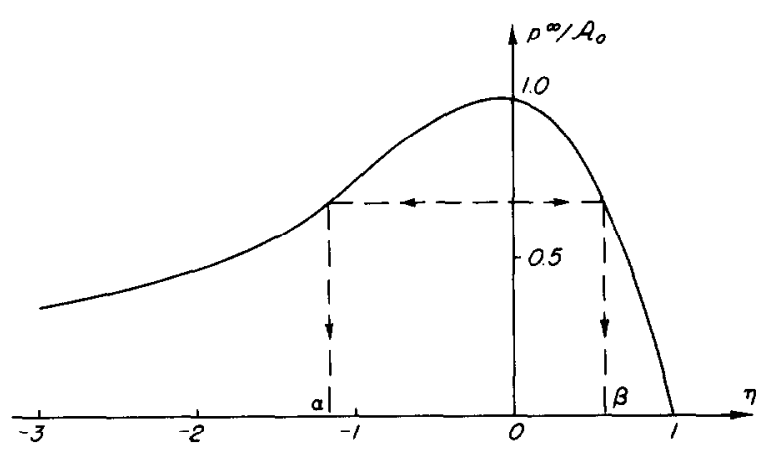

Fig. 2. Extent of separation for the parabolic tension pulse considered.

may be very long in comparison to the incoming disturbance. In fact, the gap does not close at all on the left if $p^{\infty}=0$.

\subsection{Square tension pulse}

Consider the incident square pulse

$$
f(\eta)=\left\{\begin{array}{lc}
1 ; & (|\eta|)<1) \\
0 ; & (|\eta|>1) .
\end{array}\right.
$$

By repeating the previous procedure we obtain

$$
\begin{aligned}
& \alpha=1-\frac{2 \mathscr{A}_{0}}{p^{\infty}}, \quad \beta=1, \\
& g(\eta)=\frac{\mathscr{A}_{0}}{\mathscr{A}_{d}} \begin{cases}\left(1-\frac{p^{\infty}}{\mathscr{A}_{0}}\right)(1-\eta) & (-1 \leqslant \eta \leqslant 1), \\
\frac{p^{\infty}}{\mathscr{A}_{0}}(\eta-\alpha) & (\alpha \leqslant \eta \leqslant-1)\end{cases}
\end{aligned}
$$

provided

$$
0 \leqslant p^{\infty} / \mathscr{A}_{0} \leqslant 1
$$

It is seen that the gap has a triangular shape. The dependence of the length of the separation zone on $p / \mathscr{A}_{0}$ is shown in Fig. 3.

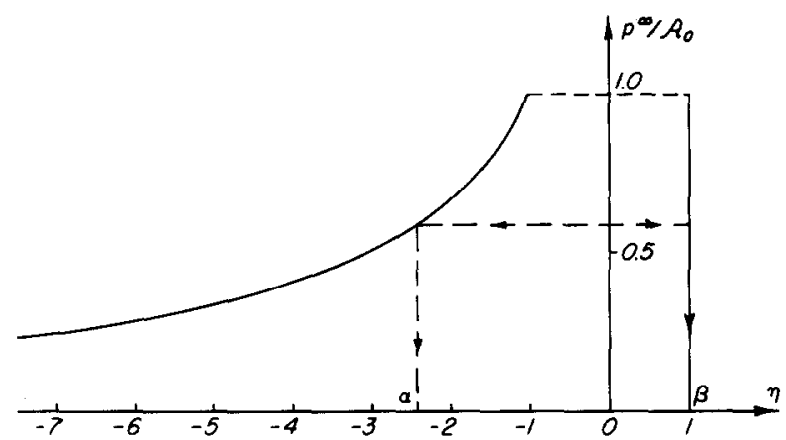

Fig. 3. Extent of separation for a square tension pulse.

\subsection{Other tension pulses}

It is clear from the previous examples as well as the general formulas that the procedure for finding the extents of separation and the resulting gaps is quite simple. Indeed, the relation

$$
\frac{\mathrm{d} g(\eta)}{\mathrm{d} \eta}=-\frac{1}{\mathscr{A}_{d}}\left[S(\eta)-p^{\infty}\right]
$$
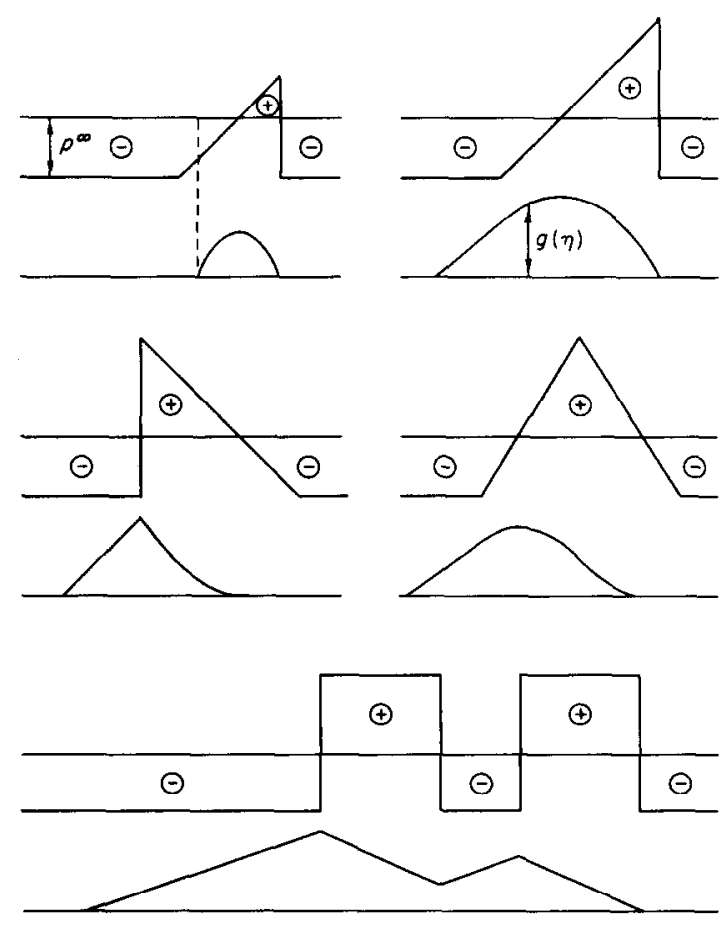

Fig. 4. Gaps resulting from various shapes of triangular and square tension pulses in the precompressed solids. The upper lines depict schematically $S(\eta)-p^{\infty}$, the lower $g(\eta)$. 
allows one to construct the gap by starting at its leading edge, proceeding to the left and simply accounting for the accumulated area under the curve $S(\eta)-p^{\infty}$ (tractions in the bilateral problem with precompression). We do not think it necessary, however, to elaborate on this point. The gaps resulting from various shapes of triangular pulses are shown schematically in Fig. 4. Included in this figure are also two square pulses that interact leading to a long gap with a broken profile.

\section{Conclusion}

An interesting feature of the results is that the gaps do not generally close smoothly at their trailing ends. Consequently the interface tractions are discontinuous at these points. This is due to the fact that the solids impact as they come back into contact [1]. In contrast, the gaps open smoothly at their leading edges, unless the incident wave involves a jump discontinuity, such as was encountered for the square tension pulse.

As the problem considered involves mixed boundary conditions and inequality constraints, it might be surprising that the solution assumed an elementary form. This is entirely due to the fact, however, that we restricted the investigation to the case when the gaps propagate along the interface with a supersonic speed. If the angle of incidence is such that the speed falls in the transonic or even subsonic ranges and total reflection is encountered, the situation becomes considerably more complicated. These aspects of the interface separation will be considered in later publications.

\section{Acknowledgment}

One of the authors (M.C.) gratefully acknowledges the support through the National Science Foundation grant (ENG-77-25032) during the course of this research.

\section{References}

[1] M. Comninou an'd J. Dundurs, "Reflection and refraction of elastic waves in presence of separation", Proceedings of the Royal Society of London A356 (1977) 509-528.

[2] M. Comninou and J. Dundurs, "Singular reflection and refraction of elastic waves due to separation", Joumal of Applied Mechanics, to appear.

[3] F.G. Friedlander, "On the total reflection of plane waves", The Quarterly Journal of Mechanics and Applied Mathematics 1 (1948) 376-384.

[4] J.D. Achenbach, Wave Propagation in Elastic Solids, North-Holland Publishing Company, Amsterdam (1973).

[5] H.M. Berg, J.E. Bloom, H. Ishii, R.H. Marion, D.E. Pease, D.T. Spreng, J.B. VanderSande and J. Weertman, "Subsonic, supersonic, and transonic dislocations moving on an interface separating two media of differing elastic properties", in: Fundamental Aspects of Dislocation Theory, edited by J. A. Simmons, R. de Wit and R. Bullough, National Bureau of Standards Special Publication 317 I (1970) 71-82.

[6] J. Weertman, "Uniformly moving transonic and supersonic dislocations", Journal of Applied Physics 38 (1967) 52935301.

[7] J. Weertman, "Dislocations in uniform motion on slip or climb planes having periodic force laws", in Mathematical Theory of Dislocations, edited by T. Mura, The American Society of Mechanical Engineers, New York (1969) 178202. 